\title{
Active silicon microring resonators using metal-oxide-semiconductor capacitors
}

Chao Li and Andrew W. Poon

Department of Electrical and Electronic Engineering, The Hong Kong University of Science and Technology, Clear Water Bay, Hong Kong SAR, China

Tel: (852)-2358-7905,fax: (852)-2358-1485, email: eeawpoon@ust.hk

\begin{abstract}
We propose an injection-type active silicon microring resonator using metal-oxide-semiconductor capacitors. Our simulations reveal a modulation bandwidth exceeding $2 \mathrm{GHz}$, a resonance shift of $0.5 \mathrm{~nm}$, and an extinction ratio exceeding $20 \mathrm{~dB}$.
\end{abstract}

OCIS codes: (230.5750) Resonators; (230.4110) Modulators

Most recently, silicon-on-insulator (SOI) electro-optical modulators with a bandwidth exceeding $1 \mathrm{GHz}$ has been experimentally demonstrated using metal-oxide-semiconductor (MOS) capacitor phase shifters on a Mach-Zehnder interferometer [1]. The carrier injection by means of MOS capacitors enables capacitor-limited high-speed and ultra low-power operation. This pioneering work is expected to open up many new $\mathrm{R} \& \mathrm{D}$ directions for silicon-based photonics.

In this summary, we propose SOI waveguide-coupled microring resonator modulators and tunable filters using MOS capacitors. The principle is that the lightwave circulating in the microring resonator and spatially overlaps with the MOS gate-oxide region can experience a phase shift due to the free-carrier dispersion effect [2,3] and result in a resonance blueshift. Active microresonator devices [4] have the key advantages of (i) compact size in the order of $10 \mu \mathrm{m}-50 \mu \mathrm{m}$, and (ii) highly wavelength selective due to whispering-gallery resonances.
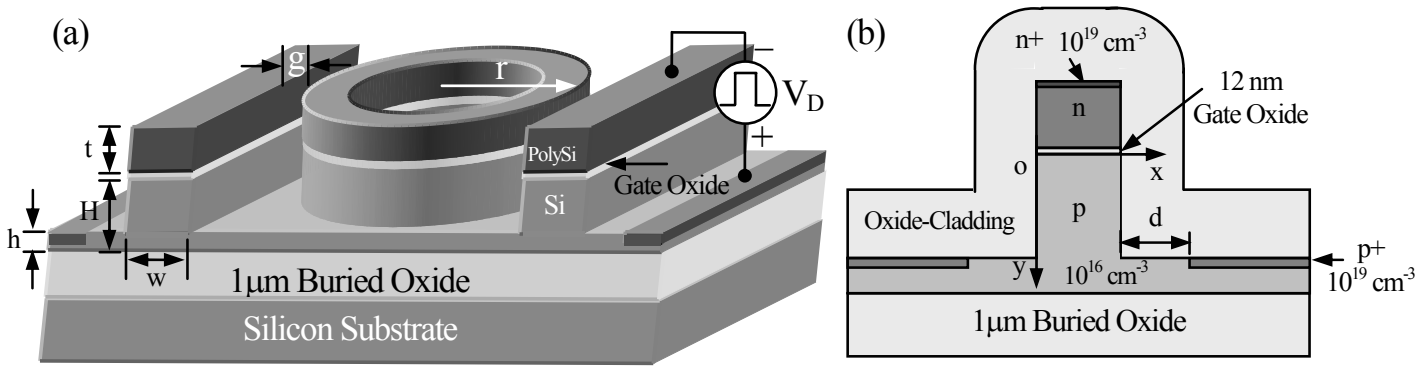

Fig. 1. (a) Schematic of an active SOI waveguide-coupled microring resonator using MOS capacitors. $\quad \mathrm{V}_{\mathrm{D}}$ is the driving voltage. $\quad r=5$ $\mu \mathrm{m}, w=0.35 \mu \mathrm{m}, g=0.35 \mu \mathrm{m}, t=0.2 \mu \mathrm{m}, H=0.34 \mu \mathrm{m}, h=0.1 \mu \mathrm{m} . \quad$ (b) Schematic cross section of the active waveguide using a MOS capacitor. $d=0.5 \mu \mathrm{m}$.

Figures 1 (a) and 1 (b) show the device schematic and the schematic cross section of the active waveguide. The device parameters are detailed in the figure caption. We adopted conventional laterally waveguide-coupled microring resonator add-drop filter configuration. For our initial device simulations, the entire input and output-coupled waveguides and the microring have the same MOS structure. The MOS structure is comprised of a 12-nm thin gate oxide [1] sandwiched between the upper n-type polysilicon layer and the p-type $\left(10^{16} \mathrm{~cm}^{-3}\right)$ silicon layer. In order to make ohmic contact to the aluminum contact, the polysilicon and the silicon slab have a surface doping concentration of $10^{19} \mathrm{~cm}^{-3}$. The entire device is oxide clad (not shown in Fig. 1 (a)).

In order to study the DC and the transient behavior of the device, we employed a commercially available two-dimensional (2-D) device simulator MEDICI. The suitability of using MEDICI in analyzing electro-optic effects in SOI waveguide-based devices has been verified [5]. From the calculated electron and hole concentration values, the free-carrier induced variation in real refractive index $\Delta \mathrm{n}$ and in absorption coefficient $\Delta \alpha$ at a wavelength of $1.55 \mu \mathrm{m}$ can be obtained [3]. For our initial calculations, we assumed the polysilicon and the single-crystal silicon have the same free-carrier dispersion and absorption effects. 

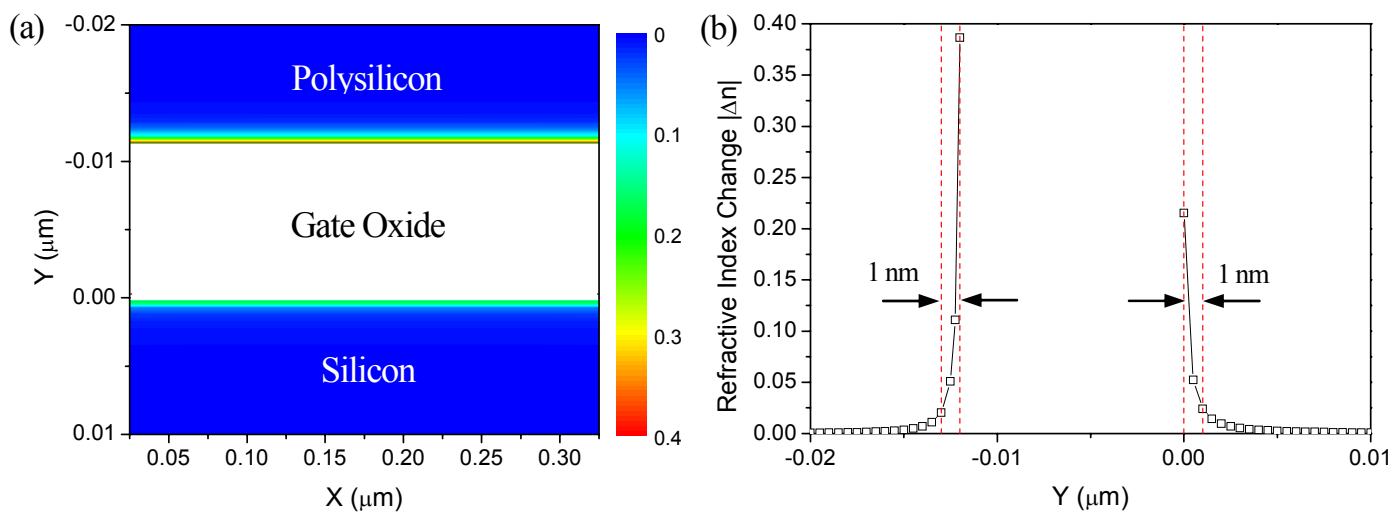

Fig. 2. (a) Contour of the absolute refractive index change in the region on both sides of the gate oxide. (b) Absolute refractive index change across the line in the $y$-direction at the center of the waveguide region $(x=0.175 \mu \mathrm{m})$.

Figure 2 (a) shows the contour of the absolute refractive index change $|\Delta \mathrm{n}|$ in the silicon and the polysilicon region on both sides of the gate oxide upon a driving voltage of $9 \mathrm{~V}$. The $|\Delta \mathrm{n}|$ in the $\mathrm{x}$-direction (away from the vicinity of the sidewalls) is uniform. Figure 2 (b) shows the $|\Delta \mathrm{n}|$ in the $\mathrm{y}$-direction along the vertical line $\mathrm{x}=0.175 \mu \mathrm{m}$ at the center of the waveguide. Note that $\Delta \mathrm{n}$ only occurs within a thin charge accumulation layer of about $1 \mathrm{~nm}$ on both sides of the gate oxide. The maximum $|\Delta \mathrm{n}|$ is about 0.39 on the polysilicon side, and is about 0.22 on the silicon side.
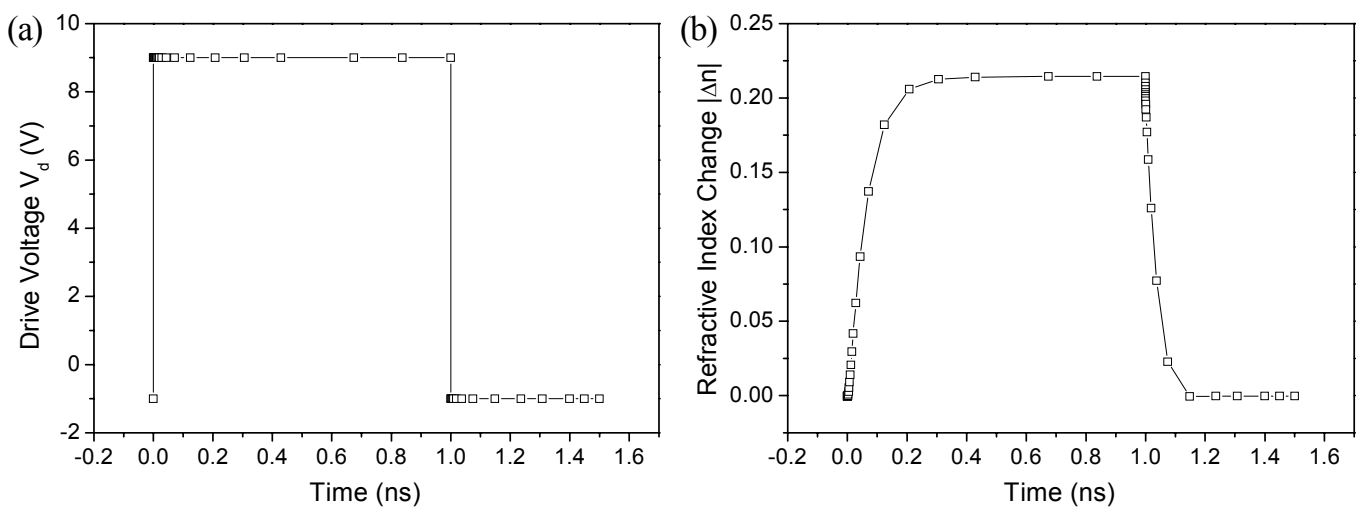

Fig. 3. (a) Driving voltage pulse with $V_{\text {on }}=9 \mathrm{~V}$ and $V_{\text {off }}=-1 \mathrm{~V}$. $\quad$ (b) Transient behavior of the absolute refractive index change $|\Delta n|$ at the gate oxide-silicon interface $(\mathrm{y}=0 \mu \mathrm{m})$ for a step driving voltage pulse in (a).

Figures 3 (a) and 3 (b) show the simulated driving voltage pulse and the free-carrier induced $|\Delta \mathrm{n}|$ evolution in time at the gate oxide-silicon interface $(\mathrm{y}=0 \mu \mathrm{m})$. The device was initially forward biased to $9 \mathrm{~V}$ (on state) and followed by a reverse bias of $-1 \mathrm{~V}$ (off state) after $1 \mathrm{~ns}$. We obtained the $10 \%$-to- $90 \%$ rise time to be $0.15 \mathrm{~ns}$ and the fall time to be $0.08 \mathrm{~ns}$. The 3-dB bandwidth therefore exceeds $2 \mathrm{GHz}$.

In order to estimate the effective refractive index $\left(\mathrm{n}_{\text {eff }}\right)$ variation of the waveguide mode upon carrier injection, we assumed $\Delta \mathrm{n}=-0.11$ (half of the maximum $\Delta \mathrm{n}$ on the silicon side) for a $1 \mathrm{~nm}$ region on both sides of the gate oxide. We employed the commercially available full-vector beam propagation method (BPM) simulation tool [6] to calculate the $\mathrm{n}_{\text {eff }}$ and the guided mode-field profile. We initially adopted a 1-nm grid size in order to simulate the 1-nm thin $\Delta \mathrm{n}$ region within a reasonable computation time. Figure 4 (a) shows the BPM simulated mode-field profile at the on state. The device parameters are detailed in Fig. 1 caption. The wavelength is $1.55 \mu \mathrm{m}$. The polarization is TM $(\mathbf{E} \perp$ plane). Inset (i) shows the zoom-in view of the strongly localized mode-field profile within the 12-nm gate oxide region. The confined mode field has a Gaussian distribution in the lateral direction and a flat top distribution in the vertical direction.

We find that the $\mathrm{n}_{\mathrm{eff}}$ reduces from 2.6967 to 2.6958 (i.e. $\Delta \mathrm{n}_{\mathrm{eff}} \approx-0.001$ ) when the device is switched from an off to an on state. We also estimated the effective absorption coefficient $\left(\alpha_{\mathrm{eff}}\right)$ variation of the waveguide mode upon carrier injection. By assuming $\Delta \alpha \approx 600 \mathrm{~cm}^{-1}$ (corresponding to $\Delta \mathrm{n}=-0.11$ through Kramers-Kronig analysis [3]) for a $1 \mathrm{~nm}$ region on both sides of the gate oxide, and a relation $\Delta \mathrm{n}_{\mathrm{eff}} / \Delta \mathrm{n}=\Delta \alpha_{\mathrm{eff}} / \Delta \alpha$, we obtained $\Delta \alpha_{\mathrm{eff}} \approx 5.5 \mathrm{~cm}^{-1}$. We then calculated the imaginary part of the 
effective refractive index variation $\Delta \mathrm{n}_{\mathrm{I} \text { eff }}=\lambda \Delta \alpha_{\mathrm{eff}} / 4 \pi \approx 6.8 \times 10^{-5}$ at $1550 \mathrm{~nm}$ wavelength.

We used 2-D finite-difference time-domain (FDTD) simulations [6] to calculate the change in resonance response upon $\Delta \mathrm{n}_{\text {eff }}$ and $\Delta \mathrm{n}_{\text {Ieff. }}$. In our initial simulations, we assumed the polysilicon has the same refractive index $(\mathrm{n}=3.5)$ and losses as the single-crystal silicon. We remark that in practice polysilicon waveguides tend to have a much higher scattering loss due to the coarse polycrystalline grains than the single-crystal silicon [7].

Figure 4 (b) shows the calculated resonance blueshift of about $0.5 \mathrm{~nm}$ by imposing a $\Delta \mathrm{n}_{\text {eff }}=-0.001$ and a $\Delta \mathrm{n}_{\text {Ieff }}=6.8 \times 10^{-5}$. At the off-state (solid line), the resonance has a $3-\mathrm{dB}$ width $\approx 0.2 \mathrm{~nm}(\mathrm{Q} \approx 7,800)$ and a coupling efficiency of about $99.1 \%$. At the on-state (dashed line), the resonance has a broadened $3-\mathrm{dB}$ width $\approx 0.26 \mathrm{~nm}(\mathrm{Q} \approx 6,000)$ and a reduced coupling efficiency of about $90.8 \%$. For modulator applications, our initial simulations indicate that an extinction ratio exceeding $20 \mathrm{~dB}$ can be attained at the resonance wavelength (shown as the blue dashed line) upon a resonance shift of about $0.5 \mathrm{~nm}$. For tunable filter applications, the accompanied linewidth broadening and the reduction in coupling efficiency are potential limitations that require further optimization.
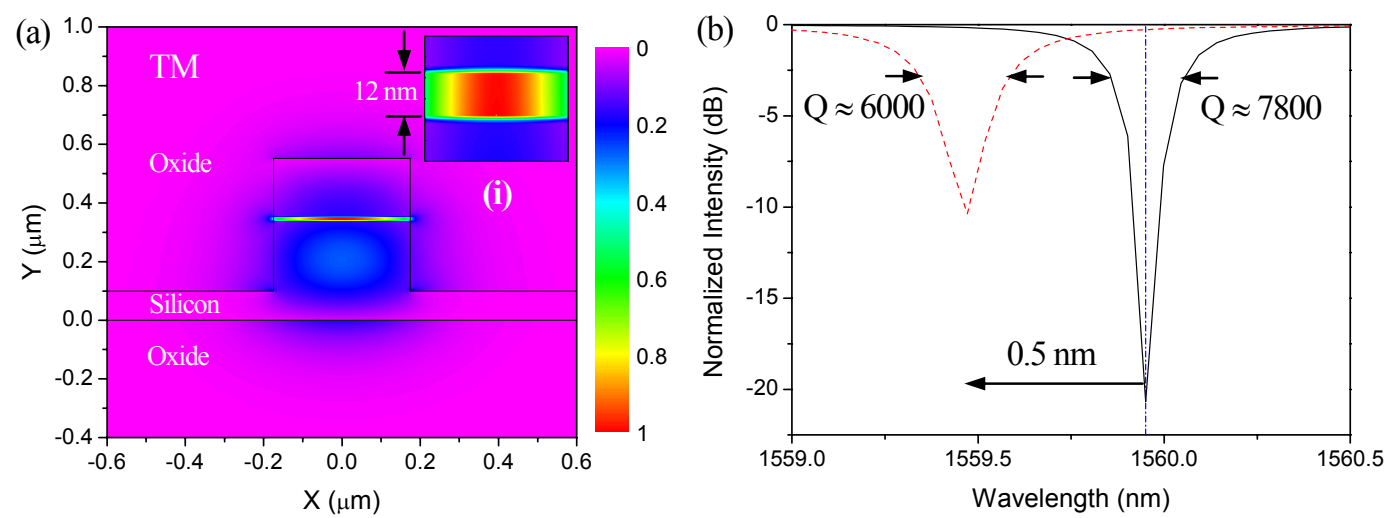

Fig. 4. (a) BPM simulated TM-polarized waveguide mode-field profile. Inset (i) shows the zoom-in view of the strongly localized mode

field inside the 12-nm thin oxide region. (b) Resonance blueshift from the off-state (solid line) to the on-state (dashed line).

In summary, we report for the first time to our knowledge, a numerical study of an injection-type active microring resonator device using MOS capacitors. Our initial simulations reveal that the capacitor-induced free-carrier dispersion effect can tune the resonance wavelength over a sub-nanometer spectral range with a $\mathrm{GHz}$ bandwidth by using about $10 \mathrm{~V}$ biasing. Further design parameter optimization and device fabrication on thin-film SOI substrates using standard CMOS processes are in progress.

We would like to acknowledge the support from the Research Grants Council and the University Grants Council of the Hong Kong Special Administrative Region, China (Project No. HKUST6166/02E \& HIA01/02.EG05), and the support from The Institute of Integrated Micro Systems (Project No. I2MS01/02.EG07).

\section{References}

[1] A. Liu, R. Jones, L. Liao, D. Samara-Rubio, D. Rubin, O. Cohen, R. Nicolaescu and M. Paniccia, "A high-speed silicon optical modulator based on a metal-oxide-semiconductor capacitor", Nature, vol. 427, pp. 615-618, 2004.

[2] R. A. Soref and B. R. Bennett, "Electrooptical effects in silicon”, IEEE J. Quant. Electron, vol. 23, pp. 123 - 129, 1987.

[3] R. A. Soref and B. R. Bennett, "Kramers-Kronig analysis of electro-optical switching in silicon”, in Proc. SPIE, vol. 704, pp. 32-37, 1986.

[4] R. Grover, T. A. Ibrahim, S. Kanakaraju, M. L. Lucas, L. C. Calhoun, P.-T. Ho, "Tunable GaInAsP-InP optical microresonator notch filter," IEEE Photonics Tech. Lett., vol. 16, pp. $467-469,2004$.

[5] G. T. Reed and A. P. Knights, Silicon Photonics (Wiley, 2004).

[6] FullWAVE, Rsoft Inc. Research Software, http://www.rsoftinc.com.

[7] A. M. Agarwal, L. Liao, J. S. Foresi, M. R. Black, X. Duan, L. C. Kimerling, "Low-loss polycrystalline silicon waveguides for silicon photonics", J. Appl. Phys., vol. 80, pp. 6120-6123, 1996. 